\title{
Exercise Reduces GABA Synaptic Input onto Nucleus Tractus Solitarii Baroreceptor Second-Order Neurons via NK1 Receptor Internalization in Spontaneously Hypertensive Rats
}

\author{
Chao-Yin Chen (陳昭吟), Andrea G. Bechtold, Jocelyn Tabor, and Ann C. Bonham \\ Department of Pharmacology, University of California, Davis, Davis, California 95616
}

\begin{abstract}
A single bout of mild to moderate exercise can lead to a postexercise decrease in blood pressure in hypertensive subjects, namely postexercise hypotension (PEH). The full expression of PEH requires a functioning baroreflex, hypertension, and activation of muscle afferents (exercise), suggesting that interactions in the neural networks regulating exercise and blood pressure result in this fall in blood pressure. The nucleus tractus solitarii (NTS) is the first brain site that receives inputs from nerves carrying blood pressure and muscle activity information, making it an ideal site for integrating cardiovascular responses to exercise. During exercise, muscle afferents excite NTS GABA neurons via substance $P$ and microinjection of a substance P-neurokinin 1 receptor (NK1-R) antagonist into the NTS attenuates PEH. The data suggest that an interaction between the substance P NK1-R and GABAergic transmission in the NTS may contribute to PEH. We performed voltage clamping on NTS baroreceptor second-order neurons in spontaneously hypertensive rats (SHRs). All animals were killed within $30 \mathrm{~min}$ and the patch-clamp recordings were performed $2-8 \mathrm{~h}$ after the sham/exercise protocol. The data showed that a single bout of exercise reduces (1) the frequency but not the amplitude of GABA spontaneous IPSCs (sIPSCs), (2) endogenous substance P influence on SIPSC frequency, and (3) sIPSC frequency response to exogenous application of substance P. Furthermore, immunofluorescence labeling in NTS show an increased substance P NK1-R internalization on GABA neurons. The data suggest that exercise-induced NK1-R internalization results in a reduced intrinsic inhibitory input to the neurons in the baroreflex pathway.
\end{abstract}

\section{Introduction}

In United States, one in three adults has hypertension, a major antecedent of stroke and heart failure (Rosamond et al., 2008). A single bout of mild to moderate exercise can lead to a postexercise decrease in blood pressure in hypertensive individuals, named postexercise hypotension (PEH), which can last for up to $13 \mathrm{~h}$ (Kenney and Seals, 1993).

The full expression of $\mathrm{PEH}$ requires a functioning baroreflex (Chandler and DiCarlo, 1997), an elevated resting blood pressure (i.e., hypertension), and activation of muscle afferents (exercise), suggesting that some interactions in the neural networks regulating exercise and blood pressure result in this fall in blood pressure. The nucleus tractus solitarii (NTS) is a pivotal point in the central network, integrating the primary baroreceptor sensory information with other inputs, including those from muscle af-

\footnotetext{
Received Sept. 15, 2008; revised Jan. 20, 2009; accepted Jan. 21, 2009.

This work was supported by National Institutes of Health Grant HL 67183 and American Heart Association Grant 0725045. SR-140333 was a generous gift from Sanofi Recherche.

Correspondence should be addressed to Chao-Yin Chen, Department of Pharmacology, University of California, Davis, Genome and Biomedical Sciences Facility Room 3510C, One Shields Avenue, Davis, CA 95616. E-mail: cych@ucdavis.edu.

D0I:10.1523/JNEUROSCI.4413-08.2009

Copyright $\odot 2009$ Society for Neuroscience $\quad$ 0270-6474/09/292754-08\$15.00/0
}

ferent fibers, making it an ideal site for integrating cardiovascular responses to exercise (Toney and Mifflin, 1995; Potts, 2001).

Within the NTS, GABAergic inhibition plays an important role in baroreceptor signal processing. Activation of the baroreceptor afferents often elicits an inhibitory response following the initial excitatory response in NTS, either by changing the postsynaptic membrane potential or spiking discharge of the postsynaptic NTS neurons (Mifflin, 2001). Most of the NTS neurons receive tonic GABA inputs from either interneurons in the NTS or projections from other brain regions (Mifflin, 2001). There is considerable evidence suggesting increased GABAergic inhibition in the NTS contributes to the development of hypertension (Catelli and Sved, 1988; Tsukamoto and Sved, 1993; Mifflin, 2001; Zhang et al., 2007). Thus, manipulations resulting in changes in endogenous GABA release in the NTS, such as exercise, could have a greater effect on blood pressure regulation in hypertension. However, we have no information on whether alterations in GABA mechanisms contribute to $\mathrm{PEH}$.

There is evidence that a neuropeptide, substance $\mathrm{P}$, acting on neurokinin 1 receptor (NK1-R) in the NTS, contributes to PEH. Microinjection of a substance P-NK1-R antagonist in the NTS immediately before exercise attenuates the development of $\mathrm{PEH}$ in spontaneously hypertensive rats (SHRs) (Chen et al., 2002). Several lines of evidence suggest that muscle afferent fibers release 

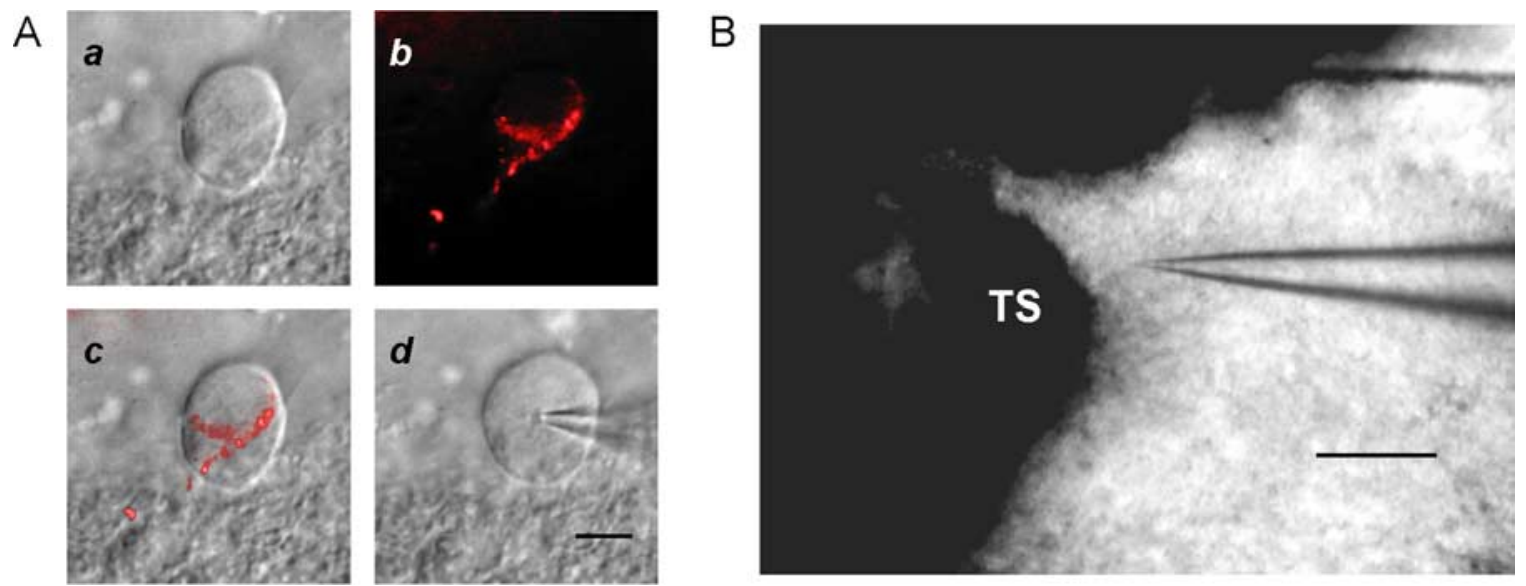

Figure 1. $\quad \boldsymbol{A}$, An NTS baroreceptor second-order neuron. $\boldsymbol{a}$, The neuron viewed with infrared differential interference contrast (IR-DIC). $\boldsymbol{b}$, The labeled aortic depressor nerve boutons viewed with fluorescence filter set. $\boldsymbol{c}$, Overlay of the IR-DIC and fluorescence images. $\boldsymbol{d}$, Neuron with patch electrode in whole-cell configuration. Scale bar, $10 \mu \mathrm{m}$. $\boldsymbol{B}$, Photomicrograph shows the position of the recording electrode. TS, Tractus solitarius. Scale bar, $200 \mu \mathrm{m}$.

A

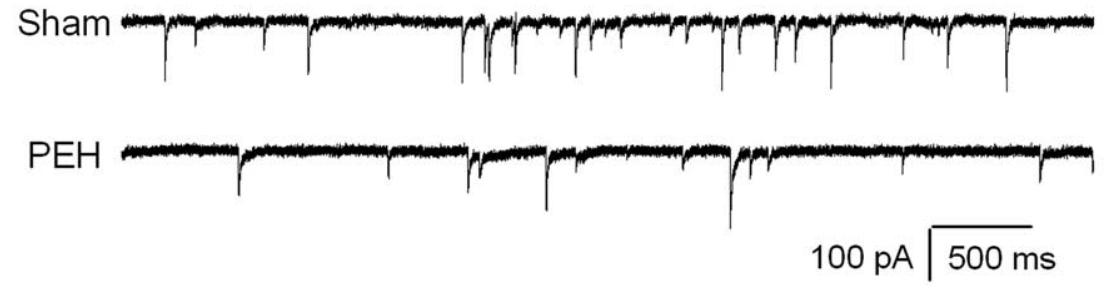

B
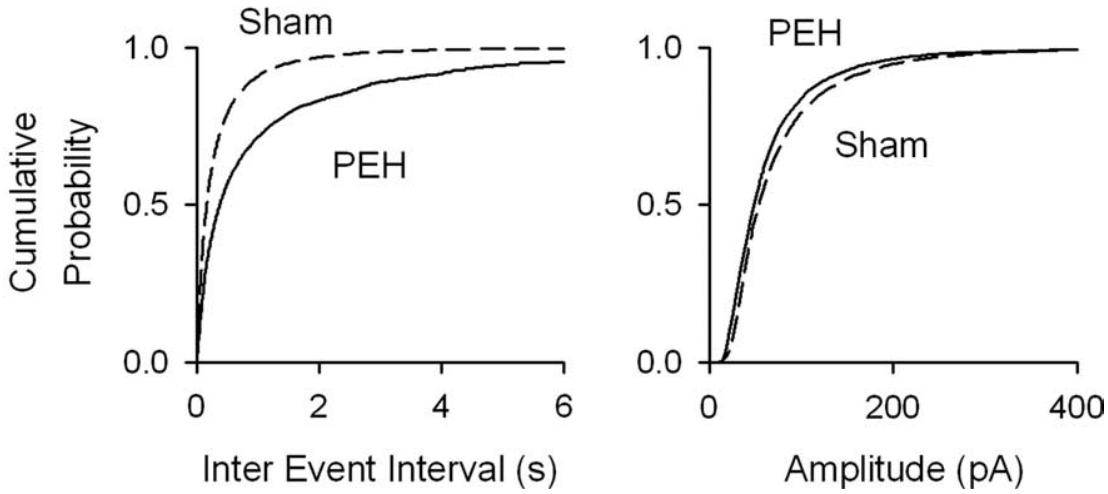

Figure 2. $\quad \boldsymbol{A}$, Example traces of sIPSC recorded from one Sham and one PEH SHR. $\boldsymbol{B}$, Group data of cumulative probability of sIPSC interevent interval (left) and amplitude (right). PEH was associated with a rightward shift in the interevent interval and reduced mean frequency. There was no change in the sIPSC amplitude after exercise.

substance $\mathrm{P}$ to activate NK1-R on GABA neurons in the NTS to modify baroreflex function during exercise (Potts et al., 1999; Boscan and Paton, 2005; Potts, 2006). The data raised the possibility that the unique interaction between the substance $\mathrm{P}$ and GABAergic signal transmission systems may contribute to $\mathrm{PEH}$. Since activation of the NK1-R has been shown to result in the receptor internalization (Marvizón et al., 2003), exercise-induced substance P NK1-R internalization on GABA neurons may provide the unique interaction between the two neurotransmission systems to trigger $\mathrm{PEH}$.

We propose to test the hypothesis that a single bout of dynamic exercise decreases the GABA inhibitory synaptic inputs to the NTS baroreceptor second-order neurons via substance $\mathrm{P}$ NK1-R internalization on GABA neurons in SHRs.

\section{Materials and Methods}

All experimental protocols in this work were reviewed and approved by the Institutional Animal Care and Use Committee in compliance with the Animal Welfare Act and in accordance with Public Health Service Policy on Humane Care and Use of Laboratory Animals.

Surgical preparation for labeling aortic depressor nerve boutons. Male spontaneously hypertensive rats (SHRs) (12 weeks old, 220-325 g; Charles River Laboratories) were anesthetized with a combination of ketamine $(50 \mathrm{mg} / \mathrm{kg}$ ) and xylazine $(8 \mathrm{mg} / \mathrm{kg})$. The criteria for adequacy of anesthesia include (1) lack of eye blink reflex, (2) no whisker movement, (3) lack of paw withdrawal in response to pinch, and (4) no irregular or sudden changes in breathing frequency. The aortic depressor nerve (ADN) was labeled with a fluorescent dye, 1,1'-dilinoleyl3,3,3',3'-tetramethylindocarbocyanine, 4-chlorobenzenesulfonate [FAST DiI solid; DiI $\Delta^{9,12}$. $\mathrm{C}_{18}(3)$ ] as previously described (Bonham and Chen, 2002; Chen and Bonham, 2005; Sekizawa and Bonham, 2006).

Exercise protocol. During the second week of recovery from surgery, all rats were acclimated to the treadmill by placing them on the treadmill for 40 min daily. On the day of the experiment, the rats were randomly assigned to an exercise group (PEH) that were subjected to a single bout of exercise on the motor driven treadmill at $15-16 \mathrm{~m} / \mathrm{min}$ and $10^{\circ}$ for $40 \mathrm{~min}$ or to a sham-exercise group (Sham), placed on the treadmill for $40 \mathrm{~min}$ with no exercise. All animals were killed within $30 \mathrm{~min}$ after the end of sham/ exercise protocol.

Brainstem slice preparation. After the sham or exercise protocol, the rats were anesthetized with a combination of ketamine $(50 \mathrm{mg} / \mathrm{kg})$ and xylazine $(8 \mathrm{mg} / \mathrm{kg}$ ) and decapitated. The brain slices containing the NTS were obtained as previously described (Bonham and Chen, 2002; Chen and Bonham, 2005; Sekizawa and Bonham, 2006). During the experiments a single slice was transferred to the recording chamber, held in place with a nylon mesh, and continuously perfused with oxygenated aCSF at a rate of $\sim 3 \mathrm{ml} / \mathrm{min}$. All electrophysiological recordings were performed at $33-34^{\circ} \mathrm{C}$ and $2-8 \mathrm{~h}$ after the end of sham/exercise protocol.

Whole-cell voltage-clamp recordings. All whole-cell voltage-clamp recordings were performed on NTS baroreceptor second-order neurons with attached fluorescent ADN boutons. The neurons were visualized 
with infrared differential interference contrast (IR-DIC) and the fluorescent boutons were visualized with an optical filter set for DiI (XF108, Omega Optical) and an image integrating system (InvestiGater, DageMTI). All images were captured with a charge-coupled device (CCD) camera (CCD-100, Dage-MTI), displayed on a TV monitor, and stored in a PC computer using Computer Eyes software (Digital Vision). Borosilicate glass electrodes were filled with a $\mathrm{CsCl}$ solution containing (in $\mathrm{mm}$ ): $140 \mathrm{CsCl}, 5 \mathrm{NaCl}, 1 \mathrm{MgCl}_{2}$, $3 \mathrm{~K}$-ATP, $0.2 \mathrm{Na}$-GTP, 10 EGTA, 10 HEPES, and 5 QX314. The $\mathrm{pH}$ was adjusted to 7.3 with $\mathrm{CsOH}$. With this pipette solution, the reversal potential for $\mathrm{Cl}^{-}$calculated from Nernst equation is $0.7 \mathrm{mV}$. The junction potential was $-3 \mathrm{mV}$ and was corrected. The seal resistance was $>1 \mathrm{G} \Omega$. The pipette resistance ranged from 2 to $5 \mathrm{M} \Omega(3.3 \pm 0.7 \mathrm{M} \Omega$, mean $\pm \mathrm{SD})$. The series resistance was no greater than $20 \mathrm{M} \Omega(13.7 \pm 3.8 \mathrm{M} \Omega$, mean $\pm \mathrm{SD})$ and not different between Sham $(13.8 \pm 3.9 \mathrm{M} \Omega$, mean $\pm \mathrm{SD})$ and $\mathrm{PEH}(13.5 \pm 3.8 \mathrm{M} \Omega$, mean \pm SD). Recordings were made with the Axoclamp 1D patch-clamp amplifier (Axon Instruments). Whole-cell currents were filtered at 2-5 $\mathrm{kHz}$ and digitized at $10 \mathrm{kHz}$ with the DigiData 1200 Interface (Axon Instruments).

The neurons were voltage clamped at $-60 \mathrm{mV}$. All experiments were performed in the presence of the ionotropic glutamate receptor antagonists, 1,2,3,4-tetrahydro-6-nitro-2,3-dioxo-benzo[f] quinoxaline-7sulfonamide disodium salt (NBQX, $10 \mu \mathrm{M})$ and DL-2-amino-5phosphonopentanoic acid $(\mathrm{AP}-5,50 \mu \mathrm{M})$ to isolate the IPSCs from EPSCs.

Electrophysiological protocols. To determine the inhibitory synaptic input to NTS baroreceptor second-order neurons during PEH, the spontaneous IPSCs (sIPSCs) were recorded for $6 \mathrm{~min}$. The sIPSCs in the last 3 min of the recording were used for data analysis. To isolate the action potential independent synaptic inputs, the miniature IPSCs (mIPSCs) were recorded in the presence of the sodium channel blocker, tetrodotoxin (TTX, $1 \mu \mathrm{M}$ ). To determine the role of the NK1-R on the inhibitory synaptic inputs, some slices were incubated with a concentration of $1 \mu \mathrm{M}$ of the substance P NK1-R antagonist (SR-140,333) for at least $1 \mathrm{~h}$ before performing the patch-clamping protocol for sIPSC and mIPSC recordings. To determine the extent of activation of substance P NK1-R on inhibitory inputs, sIPSCs and mIPSCs were recorded for 3 min during the control period, $1 \mathrm{~min}$ during perfusion with one of the concentrations of the substance $\mathrm{P}(0.01-30 \mu \mathrm{M})$ in a random order, and a recovery period (5-20 min).

To confirm that the IPSCs were GABA mediated, IPSCs were recorded (1) at different holding potentials from $-60 \mathrm{mV}$ to $+60 \mathrm{mV}$ at $30 \mathrm{mV}$ increments to determine the reversal potential; and (2) before, during, and after perfusion with the $\mathrm{GABA}_{\mathrm{A}}$ receptor antagonist (bicuculline, 10 $\mu \mathrm{M})$ at a holding potential of $-60 \mathrm{mV}$. To test the effectiveness of the NK1-R antagonist, the sIPSC response to $3 \mu \mathrm{M}$ substance $\mathrm{P}$ was determined with and without prior incubation with $1 \mu \mathrm{M}$ (at least for $1 \mathrm{~h}$ ) of the NK1-R antagonist, SR-140,333.

Immunofluoresence protocols. Triple-label immunohistochemistry for the NK1-R, glutamic acid decarboxylase (GAD 67, a marker for GABA neurons), and SYTOX green (nuclear counterstain) was performed in sham and PEH rats to determine whether NK1-R internalize in response to exercise.

After the sham or exercise protocol, the rats were deeply anesthetized with ketamine $(50 \mathrm{mg} / \mathrm{kg})$ and xylazine $(8 \mathrm{mg} / \mathrm{kg})$ and perfused transcardially with $200 \mathrm{ml}$ of $0.01 \mathrm{M}$ PBS (pH 7.4) followed by $500 \mathrm{ml}$ of $4 \%$ paraformaldehyde in PBS. The brain was removed, postfixed for $2 \mathrm{~h}$ in $4 \%$ paraformaldehyde, and stored overnight in PBS. Coronal brainstem slices (50 $\mu \mathrm{m}$ thick) were sectioned with a Leica VT 1000 S vibratome (Leica Microsystems). Tyramide signal amplification (TSA) immunofluorescence was performed on free-floating tissue. All incubation and rinsing steps were at room temperature on a laboratory rocker unless otherwise noted. Tissue was rinsed $3 \times 10 \mathrm{~min}$ in PBS at this point and between each subsequent step in the protocol. Sections were blocked in $1 \%$ blocking reagent (TSA kit, Invitrogen) and 10\% normal goat serum (NGS, Vector Laboratories) for $60 \mathrm{~min}$, then incubated simultaneously in primary antibodies for the NK1-R $(0.2 \mu \mathrm{g} / \mathrm{ml}$ polyclonal rabbit anti-NK1-R, Chemicon AB5897) and GAD-67 (0.5 $\mu \mathrm{g} / \mathrm{ml}$ monoclonal mouse antiGAD, Chemicon MAB5406) in PBS/1\% block/1\% NGS for 60-72 h at
A

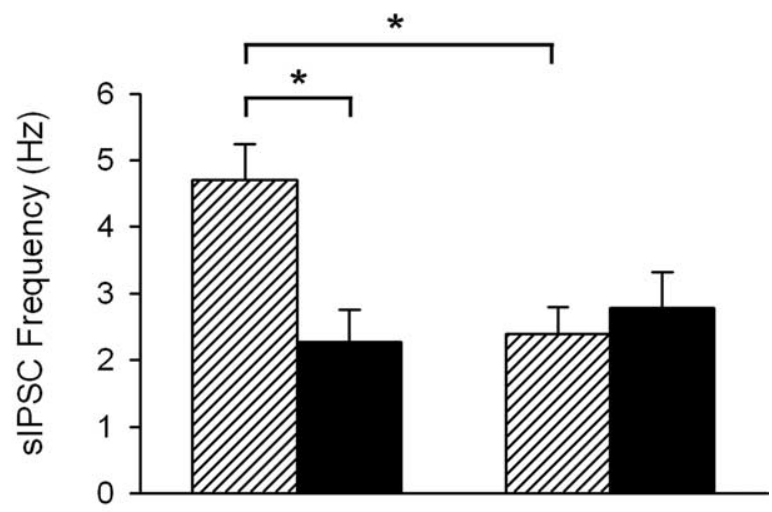

B

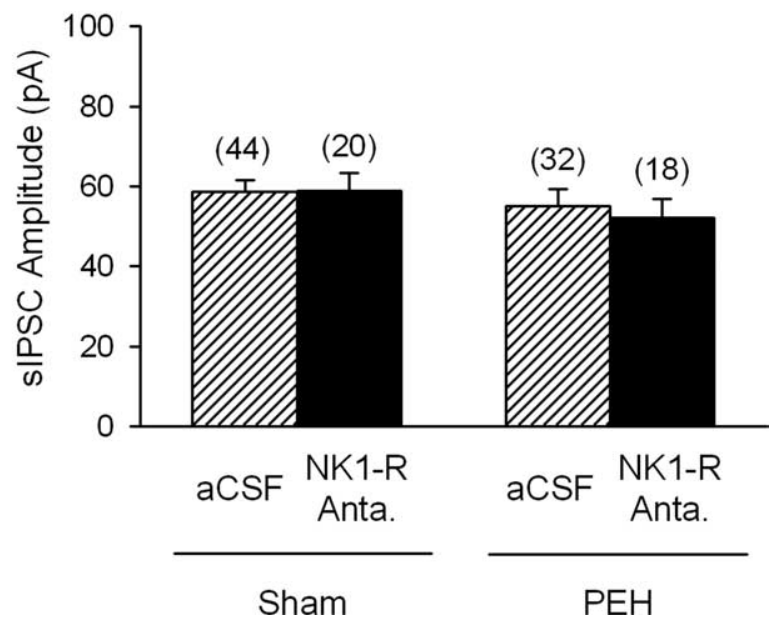

Figure 3. $\boldsymbol{A}$, Group data of sIPSC frequency. Incubation with the NK1-R antagonist (SR140,333, $1 \mu \mathrm{m}$ ) significantly reduced the sIPSC frequency in Sham but not in PEH SHRs. $\boldsymbol{B}$, Group data of sIPSC amplitude. In the same neurons, NK1-R antagonist had no effect on sIPSC amplitude in both groups. Numbers in parentheses indicate number of neurons. ${ }^{*} p<0.05$, Fisher's LSD test.

$4^{\circ} \mathrm{C}$. Sequential incubation in secondary antibodies and dye-labeled tyramide was used to detect primary antibodies. First, tissue was incubated in biotinylated goat anti-rabbit secondary antibody $(3 \mu \mathrm{g} / \mathrm{ml}$, Vector Laboratories) for $100 \mathrm{~min}$, followed by streptavidin-labeled horseradish peroxidase (2.5 $\mu \mathrm{g} / \mathrm{ml} \mathrm{SA-HRP,} \mathrm{TSA} \mathrm{kit} \mathrm{\# 23,} \mathrm{Invitrogen)} \mathrm{for} 60 \mathrm{~min}$. Staining was visualized with Alexa Fluor 546-labeled tyramide (1:100) diluted in amplification diluent $+0.0015 \%$ hydrogen peroxide for $22 \mathrm{~min}$ (TSA kit, Invitrogen). Sections were incubated in $0.3 \%$ hydrogen peroxide for $30 \mathrm{~min}$ to quench remaining peroxidase activity of the SA-HRP, rinsed, and incubated in biotinylated goat anti-mouse antibody for $100 \mathrm{~min}$, followed by SA-HRP for $60 \mathrm{~min}$. The anti-mouse secondary antibody was visualized with tyramide conjugated to an Alexa Fluor 647 (1:100 TSA kit \# 6, Invitrogen). Sections were stored overnight in PBS, then the cell nuclei were counterstained with SYTOX Green $(0.25 \mathrm{~mm})$ following incubation with RNase A $(1 \mathrm{mg} / \mathrm{ml})$ to minimize cytoplasmic staining. Sections were mounted on lysine coated slides, dried, and coverslipped in anti-fade mounting media (Vectashield, Vector Laboratories H1000). Negative controls were as follows: (1) sections run without primary or secondary antibody or SA-HRP to determine nonspecific staining due to secondary antibody, SA-HRP, or tyramide respectively; and (2) to ensure adequate destruction of HRP activity of the first SA-HRP before incubation in the second SA-HRP, some sections run without the second SAHRP were reacted with Alexa Fluor 647 tyramide following treatment with hydrogen peroxide.

Data analysis. The IPSC events were detected with Mini Analysis software (Synaptosoft). The accuracy of detection was confirmed by visual inspection. Data are expressed as means \pm SE unless otherwise indicated. Differences were considered significant at $p<0.05$. The statistical anal- 
A Control 3 $3 \mu \mathrm{M}$ Substance $\mathrm{P}$ Sham

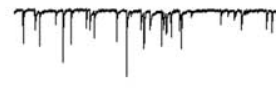
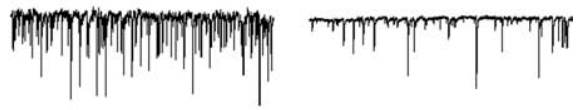

\section{$\mathrm{PEH}$}
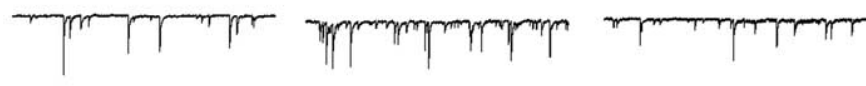

$$
2 0 0 \mathrm { pA } \longdiv { 1 \mathrm { s } }
$$

B

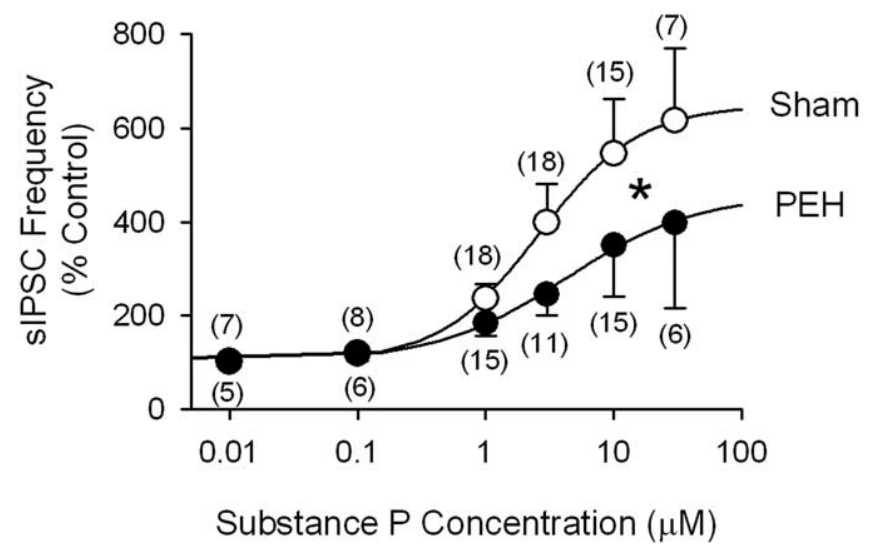

Figure 4. $\quad A$, Example traces of sIPSCs recorded before (Control), during substance $P$ perfusion, and during washout period from one Sham and one PEH SHR. $\boldsymbol{B}$, Group data of substance P-induced increase in sIPSC frequency. Substance P concentrationdependently increased sIPSC frequency in baroreceptor second-order neurons. The neurons recorded from PEH rats had a small response to application of substance P. Numbers in parentheses indicate number of neurons. ${ }^{*} p<0.05$, two-way ANOVA.

\section{A}

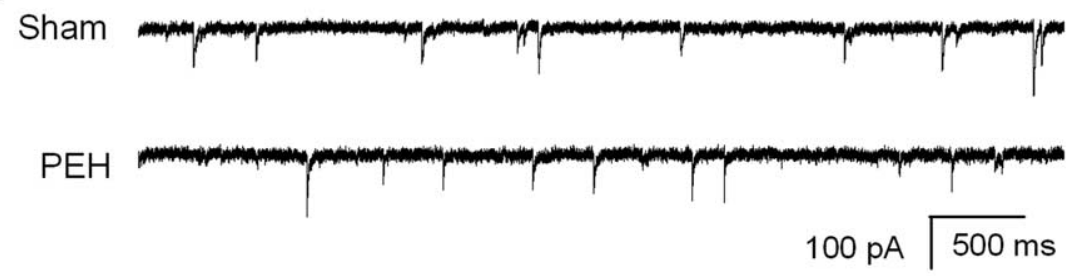

B

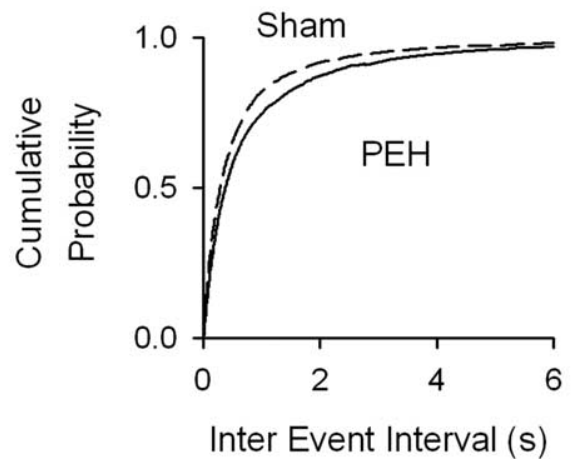

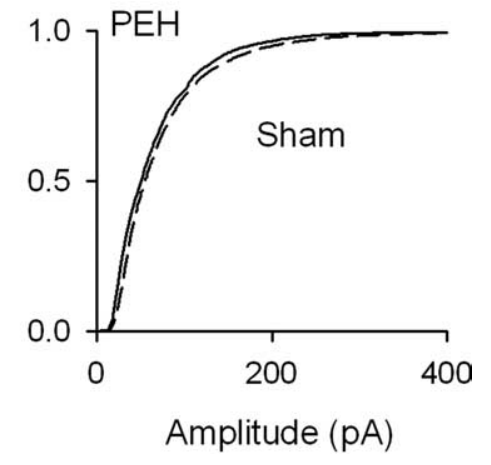

Figure 5. A, Example traces of mIPSC recorded from one Sham and one PEH SHR. $\boldsymbol{B}$, Group data showing no difference in cumulative probability of interevent interval (left) and amplitude (right).

Sham/PEH as one factor and substance $\mathrm{P}$ doses as the other factor. The sIPSC frequency response to $3 \mu \mathrm{M}$ substance $\mathrm{P}$ in the presence and absence of NK1-R antagonist was compared with a $t$ test. The MIPSC data were analyzed the same way as sIPSCs.

For immunofluorescence of the brain tissue, slices were viewed and images were captured with a Zeiss LSM-5 inverted laser scanning confocal microscope. The imaging and data analysis were performed in a blinded manner. Four hemislices corresponding to the NTS region for whole-cell patch-clamp recordings were selected for analysis: one hemislice at the level of caudal NTS, two at the level of area postrema, and one at the level of rostral NTS. Slices were scanned sequentially with a $488 \mathrm{~nm}$ (SYTOX green), $543 \mathrm{~nm}$ (NK1-R), and $633 \mathrm{~nm}$ (GAD67) laser lines under a $40 \times$ oil objective. Images were scanned and captured at a resolution of $1024 \times 1024$ or $1564 \times 1564$ with $1 \mu \mathrm{m}$ steps. Only neurons with double labeling of NK1-R and GAD 67 were counted. In addition to visual classification of the neurons with internalized substance P NK1-R, the fluorescence intensity for NK1-R and GAD 67 were measured and plotted using the ImageJ software (W. S. Rasband, ImageJ, United States National Institutes of Health, Bethesda, MD, USA, http://rsb.info. nih.gov/ij/, 1997-2007). The neurons with peak NK1-R fluorescent intensity outside of the GAD 67 fluorescent intensity were classified as having surface NK1-R. The neurons with overlapping distribution for NK1-R and GAD 67 were classified as having internalized NK1-R. The ratio of neurons with internalized NK1 receptors to neurons with surface NK1-R was calculated for each animal and compared with a $t$ test.

Drugs. Ketamine and xylazine were obtained from Vedco. DiI and TSA kits were obtained from Molecular Probes. RNase A and SYTOX Green were obtained from Invitrogen. Polyvinylsiloxane gel was obtained from Carlisle Laboratories. QX314 was obtained from Tocris. Bicuculline, Mg-ATP, Na-GTP, EGTA, and HEPES were obtained from Sigma. All other chemicals were obtained from Fisher. SR140333 was a generous gift from Sanofi Recherche.

\section{Results}

All electrophysiological data were obtained in NTS baroreceptor second-order neurons identified by their possession of fluorescently labeled attached boutons as shown in Figure $1 A$, located in the dorsal and medial NTS between $250 \mu \mathrm{m}$ rostral to obex and $250 \mu \mathrm{m}$ caudal to calamus scriptorius (Fig. 1B).

yses were performed with SigmaStat software (SPSS). When appropriate, the ANOVA test was followed by Fisher's LSD test for pairwise comparisons. A $t$ test was used to compare the sIPSC frequency and amplitude between Sham and PEH group. A two-way ANOVA was used for comparing the sIPSC frequency and amplitude with and without NK1-R antagonist with Sham/PEH as one factor and incubation with NK1-R antagonist as the other factor. A two-way ANOVA was also used to compare the sIPSC frequency responses to substance P perfusion with
Exercise decreased inhibitory input to NTS baroreceptor second-order neurons via substance P NK1-R mechanisms A single bout of dynamic exercise decreased the frequency but not the amplitude of sIPSCs (Fig. 2). Example recordings of sIP$\mathrm{SC}$ from one Sham and one $\mathrm{PEH}$ rat are shown in Figure $2 \mathrm{~A}$. The group data showed a rightward shift in the cumulative probability of the sIPSC interevent intervals (Fig. $2 B$, left). The mean 
frequency was significantly lower $(t$ test, $p<0.05)$ in $\mathrm{PEH}(2.4 \pm$ $0.4 \mathrm{~Hz}, n=44)$ compare with Sham group $(4.7 \pm 0.5 \mathrm{~Hz}, n=32)$. There was no change in the cumulative probability of the sIPSC amplitude (Fig. $2 \mathrm{~B}$, right). The median amplitude was not different $(t$ test, $p>0.05)$ between Sham $(59 \pm 3 \mathrm{pA})$ and PEH (55 \pm $4 \mathrm{pA})$. The data suggest that there is a reduced inhibitory input to the NTS baroreceptor second-order neurons in PEH.

Incubation with the substance P NK1-R antagonist $(1 \mu \mathrm{M}$, SR-140,333) had no effect on sIPSC frequency in the PEH group, but significantly decreased the sIPSC frequency in the Sham group (two-way ANOVA, $p<0.05$, interaction) (Fig. $3 A$ ). The substance P NK1-R antagonist had no effect on the sIPSC amplitude in either the Sham or $\mathrm{PEH}$ groups (Fig. 3B, two-way ANOVA, $p>0.05$ ). The data suggest that the decrease in inhibitory inputs to baroreceptor second-order neurons involves substance P NK1-R mechanism(s), either a presynaptic change in substance $\mathrm{P}$ release or a postsynaptic change in NK1-R.

\section{Exercise decreased substance P NK1-R responsiveness to exogenous substance $P$}

As shown in the example traces in Figure 4, exogenous application of substance $\mathrm{P}$ elicited a significantly greater increase in sIPSC frequency in the Sham group compared with the $\mathrm{PEH}$ group (Fig. $4 A$ ). The group data confirm that while substance $\mathrm{P}$ evoked concentration-dependent increases in sIPSC frequencies in both groups, the neurons from the $\mathrm{PEH}$ rats displayed much smaller responses (Fig. 4B) (two-way ANOVA, $p<0.05$ exercise effect and dose effect). The effect of exogenous substance P (3 $\mu \mathrm{M})$ was blocked ( $t$ test, $p<0.05$ ) by incubation with the NK1-R antagonist, SR140,333 (399 \pm 82 and $146 \pm 28 \%$, without and with NK1-R antagonist, respectively). The data suggest that a reduced NK1-R function, as indicated by a reduced responsiveness to substance $\mathrm{P}$, contributes to the decrease in inhibitory inputs to baroreceptor second-order neurons during $\mathrm{PEH}$.

\section{Substance P NK1-R on cell soma/dendrites versus on terminals}

The reduced sIPSC frequency responsiveness to substance $\mathrm{P}$ could be mediated by the NK1-R located on cell soma or dendrites of the GABA interneurons to change the action potentialmediated neurotransmitter release and/or on the terminals to change the spontaneous neurotransmitter release. Since the sIPSCs recorded on baroreceptor second-order neurons contain both action potential-dependent and independent events, the miniature inhibitory events were recorded in the presence of TTX. A single bout of dynamic exercise had no significant effect on the frequency and amplitude of mIPSCs (Fig. 5). Example recording of mIPSCs from one Sham and one $\mathrm{PEH}$ rat are shown in Figure $5 A$. There was no significant difference in the cumulative probability of the interevent interval (Fig. $5 B$, left) and amplitude (Fig. 5B, right). The mean frequency was not different $(t$ test, $p>0.05)$ between Sham $(2.5 \pm 0.4 \mathrm{~Hz}, n=24)$ and $\mathrm{PEH}$ $(2.1 \pm 0.4 \mathrm{~Hz}, n=23)$, nor was the median amplitude different $(t$ test, $p>0.05 ; 57 \pm 4$ and $55 \pm 6$, Sham and $\mathrm{PEH}$, respectively). The data suggest that the reduced inhibitory input to the baroreceptor second-order neurons in NTS during PEH is mediated by action potential dependent inhibitory neurotransmitter release.

Incubation with the substance P NK1-R antagonist, SR140,333 also had no significant effect on the mIPSC frequency and amplitude in either the SHAM or PEH group (Fig. $6 A$ ). In addition, exogenous application of substance $\mathrm{P}$ failed to elicit concentration-dependent responses in either group (Fig. 6B). The results suggest that the exercise-induced reduction in inhib-
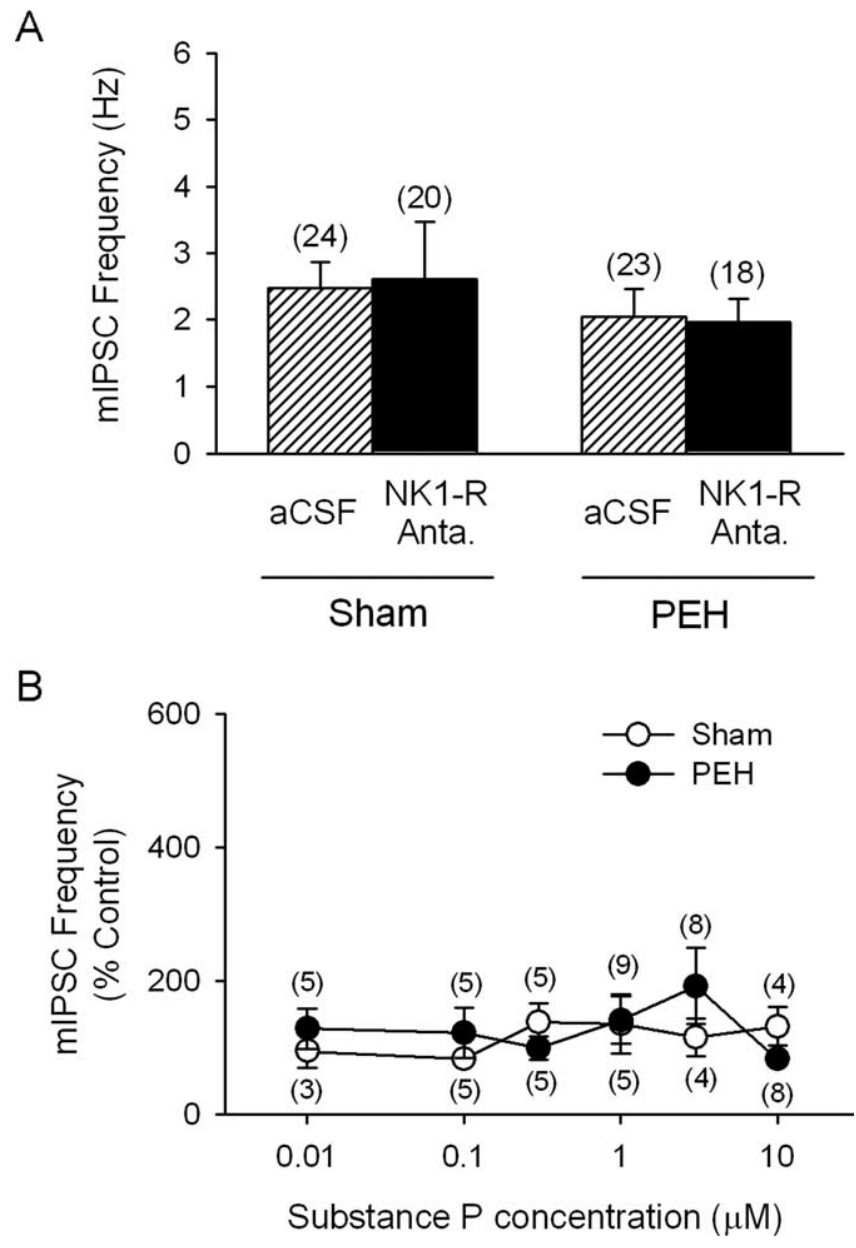

Figure 6. $\quad A$, Group data of mIPSC frequency. Incubation with the NK1-R antagonist (NK1-R Anta.) had no effect on the mIPSC frequency in both Sham and PEH groups. $\boldsymbol{B}$, Group data of effects of substance $P$ application on mIPSC frequency. Substance $P$ had no consistent effect on mIPSC frequency in baroreceptor second-order neurons. Numbers in parentheses indicate number of neurons.

itory input to the baroreceptor second-order neurons in the NTS is likely mediated by a reduced responsiveness of NK1-R located on the soma or dendrites of inhibitory neurons.

\section{The inhibitory inputs are GABAergic}

The reversal potential of the sIPSCs was $2.3 \pm 1.7$ (mean \pm SD, $n=10$ ), which is close to the calculated reversal potential for $\mathrm{Cl}^{-}$ $(0.7 \mathrm{mV})$. Bicuculline $(10 \mu \mathrm{M})$ blocked the sIPSCs in all neurons tested ( $n=20$ from Sham and $n=18$ from PEH), confirming that the inhibitory inputs were GABAergic (data not shown).

\section{Exercise induced NK1-R internalization}

For immunofluorescence study, four slices within the region used for electrophysiological recordings (between $250 \mu \mathrm{m}$ rostral to obex and $250 \mu \mathrm{m}$ caudal to calamus scriptorius) were selected for analysis: one caudal to area postrema, two at area postrema level, and one rostral to area postrema. There was no significant difference between different regions of NTS (two-way ANOVA, $p>$ 0.05 data not shown).

Figure 7 shows example images from one Sham $(A 1)$ and one PEH (A2) rat. The neuron from the Sham rat (Fig. 7A1) shows a distinct ring of NK1-R staining on the surface of the neuron. The fluorescent intensity (Fig. 7B1) plotted across the white line in Figure $7 A 1 d$ shows that the peak NK1-R fluorescent intensity was 
A1
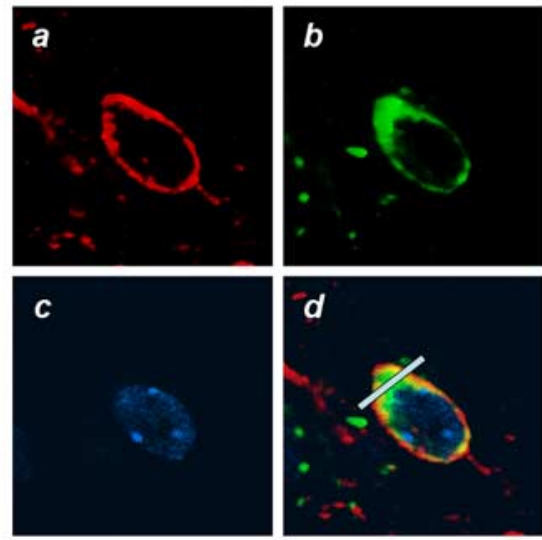

B1

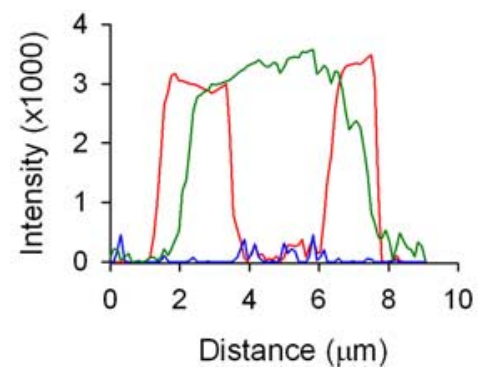

B2

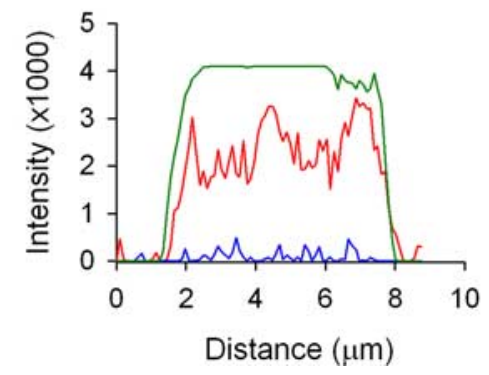

Figure 7. $\quad \boldsymbol{A}$, Example images from one Sham $(\boldsymbol{A} 1)$ and one PEH (A2) rat. $\boldsymbol{a}$, NK1-R staining (red color). $\boldsymbol{b}, \mathrm{GAD} 67$ staining (green color). c, SYTOX staining (blue color). $\boldsymbol{d}$, Overlap of $\boldsymbol{a}, \boldsymbol{b}$, and $\boldsymbol{c}$. $\boldsymbol{B}$, Fluorescent intensity plot across the white lines in $\boldsymbol{A} \mathbf{d d}$ and $\boldsymbol{A 2 \boldsymbol { d }}$. PEH was associated with a higher degree of NK1-R internalization on NTS GABA neurons.

outside of the GAD 67 fluorescent intensity confirming the surface staining of the NK1-R. The neuron from the PEH rat (Fig. $7 A 2$ ) showed a high degree of overlapping staining for NK1-R and GAD 67. The fluorescent intensity (Fig. 7B2) plotted across the white line in Figure $7 A 2 d$ showed overlapping labeling indicating NK1-R internalization. The group data confirmed that the ratio of neurons with internalized NK1-R to neurons with surface NK1-R was significantly ( $t$ test, $p<0.05$ ) higher in the PEH $(5.2 \pm 0.6, n=5)$ than in the Sham $(2.3 \pm 0.5, n=5)$ group.

\section{Discussion}

This study provides new evidence that the fall in blood pressure induced by a single bout of exercise in hypertension is mediated in part by a downregulation of NK1-R on GABA neurons synapsing onto NTS second-order baroreceptor neurons. The conclusion is based on the following findings obtained in spontaneously hypertensive rats as a model for human hypertension: (1) GABA sIPSCs recorded in baroreceptor second-order neurons displayed a decreased frequency after exercise compared with the IPSCs recorded over the same time period following Sham exercise, reflecting a decreased inhibitory GABA input during PEH; (2) the decreased GABA sIPSC frequency was accompanied by a significantly higher degree of NK1-R internalization on the GABA neurons in the PEH group compared with the Sham group, suggesting a decreased number of substance $\mathrm{P}$ binding sites on the GABA neurons after exercise; (3) the substance P NK1-R antagonist, while decreasing GABA sIPSC frequency recorded in the baroreceptor second-order neurons in the Sham group, had no effect on sIPSC frequency recorded in the $\mathrm{PEH}$ animals, indicating a blunted influence of endogenously released substance $\mathrm{P}$ binding to NK1-R on the GABA neurons in the PEH group; and (4)

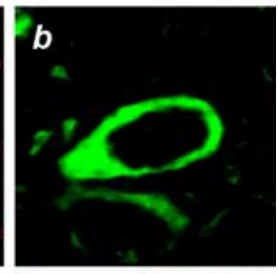

GABA sIPSC frequency following exercise was also less responsive to exogenous substance $\mathrm{P}$, compared with the Sham group, further suggesting a decreased ability of the GABA neurons to respond to substance $\mathrm{P}$ following exercise.

The expression of $\mathrm{PEH}$ requires a functioning baroreflex circuitry (Chandler and DiCarlo, 1997), hypertension, and activation of muscle afferents (exercise), so how might exercise induce NK1-R internalization in GABA neurons in the NTS and how would this contribute to PEH? Signaling in the primary baroreflex central network and how that is altered by exercise may provide some clues. In the baroreflex circuitry the primary baroreceptor afferent neurons make excitatory synapses onto second-order neurons in the NTS, which are under the inhibitory control of local GABA interneurons. The neuronal output of the NTS provides an excitatory input to neurons in the caudal ventral lateral medulla (CVLM), which in turn inhibit the activity of neurons in the rostral ventral lateral medulla (RVLM), the major output neurons controlling cardiovascular sympathetic vasomotor efferent nerve activity. Thus, with normal baroreflex function, increases in blood pressure activate the baroreceptors, which increase the activity of the NTS neurons, which ultimately results in a decreased firing of the RVLM neurons to decrease sympathetic nerve activity to return blood pressure back to control level.

Exercise resets the baroreflex to regulate blood pressure at higher blood pressures (Potts et al., 2003; Degtyarenko and Kaufman, 2005, 2006; Potts, 2006). In the current model of exerciseinduce resetting, activation of group III muscle afferent neurons blunts baroreceptor reflex mediated decrease in blood pressure during a normal blood pressure range and essentially resets the baroreflex to regulate blood pressure at higher blood pressure levels by activating NTS inhibitory interneurons (Degtyarenko and Kaufman, 2005, 2006). In addition, the exercise-induced resetting of the baroreflex can be prevented by blockade of GABA transmission in the NTS (Potts et al., 2003; Boscan and Paton, 2005). Collectively these data link exercise to activation of a local GABAergic system, which blunts the activity of NTS baroreceptor neurons to reset the operation of the baroreflex function to higher blood pressures.

The enhanced inhibitory influence to reset blood pressure during exercise appears to be mediated by the binding of substance $\mathrm{P}$, released from muscle afferent fibers, to NK1-R on GABA neurons (Potts et al., 1999; Boscan and Paton, 2005; Potts, 2006). In the present study, the internalization of NK1-R, associated with the binding to substance $\mathrm{P}$ released during exercise, reduces the effect of substance $P$ on the GABA inhibitory effects on the NTS neurons to produce PEH after exercise. This is illustrated in the model in Figure 8. During exercise substance P released from the muscle afferent fibers and subsequent activation of NK1-R on GABA interneurons triggers an increased NK1-R internalization compared with sham exercise; this NK1-R internalization is associated with a decreased responsiveness to 
the excitatory effects of substance $\mathrm{P}$ and a resultant decrease in GABA release to the baroreceptor second-order neurons. The blunting of the GABA inhibitory influence on the baroreceptor neurons has the expected downstream effects on the baroreflex signaling pathway resulting in $\mathrm{PEH}$. This model is further supported in the context of our previous study showing that $\mathrm{PEH}$ is associated with a decreased RVLM neuronal activity and sympathetic efferent nerve activity (Kajekar et al., 2002).

The decreased activity of the GABAergic system in the NTS in PEH fits into a larger picture of GABA signaling and blood pressure regulation, where previous studies have documented an exaggerated activity of the NTS GABAergic system in the development of hypertension (Catelli and Sved, 1988; Tsukamoto and Sved, 1993; Mifflin, 2001; Zhang et al., 2007). This increased GABA activity in hypertension may help to explain why exercise in hypertensive individuals is shown to consistently result in $\mathrm{PEH}$, while exercise in normotensive individuals only invariably results in $\mathrm{PEH}$ and when there is $\mathrm{PEH}$, it is smaller in magnitude (Kenney and Seals, 1993). So the more robust and reliable $\mathrm{PEH}$ in hypertensive compared with normotensive subjects is likely related to the reduced GABA release in the NTS after exercise against the background of an elevated GABA inhibition in hypertension.

A number of studies, including those from our laboratory, have previously shown that $\mathrm{PEH}$ remains robust for up to $10 \mathrm{~h}$ (Fitzgerald, 1981; Kenney and Seals, 1993; Kajekar et al., 2002). The changes in NK1-R and GABA IPSCs observed in the current study were within this $10 \mathrm{~h}$ time frame. In our previous in vivo study in conscious SHRs, which showed that PEH was accompanied by a decrease in single cardiac sympathetic neuronal activity in the RVLM as well as a decrease in efferent sympathetic vasomotor nerve activity, the recordings were made between 4 and $10 \mathrm{~h}$ (averaging $6.3 \mathrm{~h}$ ) after the exercise protocols. In the current study, the patch recordings were made between 2 and $8 \mathrm{~h}$ following sham exercise (averaging $5.22 \pm 1.27 \mathrm{~h}$ ) or exercise (averaging $5.29 \pm 1.32 \mathrm{~h}$ ), well within the time frame of the in vivo study when decreases in sympathetic activity were recorded.

The full expression of PEH is likely to be mediated by several mechanisms: from short-term to long-term changes and from peripheral nervous system to CNS effects (Kenney and Seals, 1993). In terms of central mechanisms, intracerebroventricular injections of a vasopressin V1 receptor antagonist have been shown to attenuate PEH (Collins et al., 2001). In addition, blockade of opioids with naloxone has also been shown to attenuate $\mathrm{PEH}$ (Yao et al., 1982) as well as a reduced proenkephalin gene expression in the brainstem during PEH, including the NTS (Boone and Corry, 1996). Finally, depletion of brain serotonin
SHAM

PEH
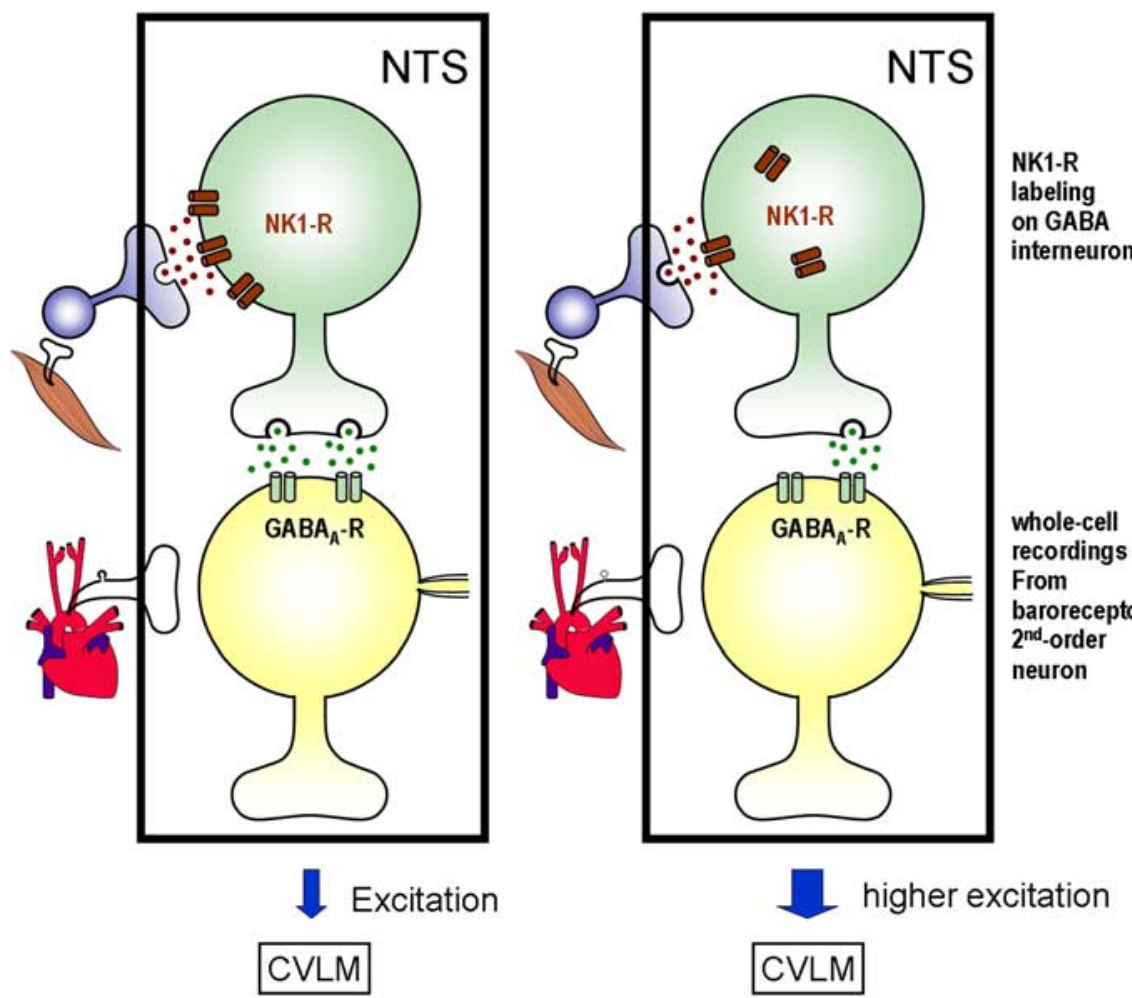

$\downarrow$ Inhibition

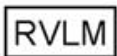

I

sympathetic activity blood pressure

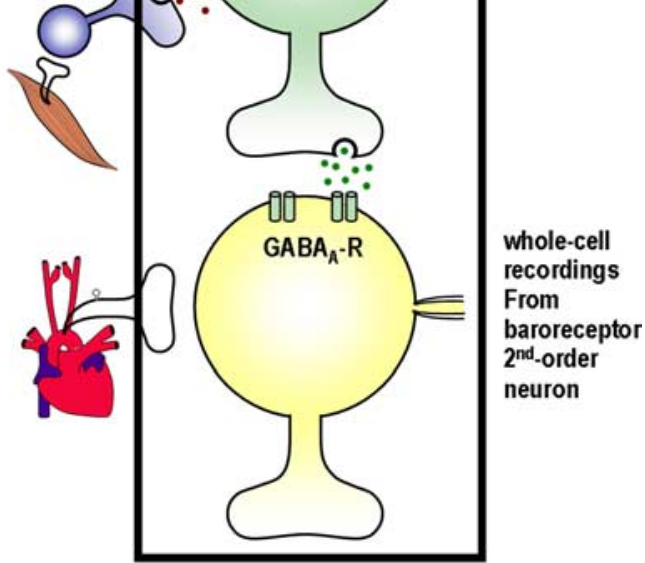

nigher excitation

CVLM

1 greater inhibition

RVLM

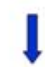

\section{Lower sympathetic activity Lower blood pressure}

Figure 8. A simplified schematic of the proposed mechanism contributing to PEH in hypertensive subjects. During exercise, effect on baroreflex regulation after exercise. Our immunofluorescence data demonstrate that the NK1-R on the NTS GABA

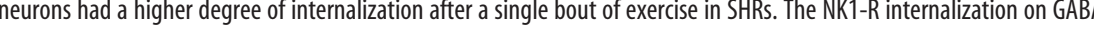
ecordings from the baroreceptor second-order neurons in NTS demonstrate that a single bout of exercise resulted in a reduced to CVLM and a greater inhibition to the RVLM resulting in a lower sympathetic activity and hence a lower blood pressure after exercise in the hypertensive subjects.

with parachlorophenylalanine has also been shown to attenuate the blood pressure lowering effect of sciatic stimulation suggesting the involvement of a central serotonergic system (Yao et al., 1982). Our data suggest that exercise-induced NK1-R downregulation to reduce GABA inhibitory input to baroreceptor secondorder neurons could provide the first line of neuroplasticity in NTS for lowering blood pressure after exercise. Collectively, the findings raise the possibility that redundant and/or interactive mechanisms from various neurotransmitter/neuromodulator systems are involved in the expression of $\mathrm{PEH}$.

In conclusion, the data are consistent with the hypothesis that a single bout of dynamic exercise decreases the GABA inhibitory synaptic inputs to the NTS baroreceptor second-order neurons via substance $\mathrm{P}$ NK1-R internalization on GABA neurons. The findings suggest that exercise-induced NK1-R downregulation 
could provide a potential novel target for lowering blood pressure in hypertensive subjects.

\section{References}

Bonham AC, Chen CY (2002) Glutamatergic neural transmission in the nucleus tractus solitarius: $N$-methyl-D-aspartate receptors. Clin Exp Pharmacol Physiol 29:497-502.

Boone JB Jr, Corry JM (1996) Proenkephalin gene expression in the brainstem regulates post-exercise hypotension. Brain Res Mol Brain Res 42:31-38.

Boscan P, Paton JF (2005) Excitatory convergence of periaqueductal gray and somatic afferents in the solitary tract nucleus: role for neurokinin 1 receptors. Am J Physiol Regul Integr Comp Physiol 288:R262-R269.

Catelli JM, Sved AF (1988) Enhanced pressor response to GABA in the nucleus tractus solitarii of the spontaneously hypertensive rat. Eur J Pharmacol 151:243-248.

Chandler MP, DiCarlo SE (1997) Sinoaortic denervation prevents postexercise reductions in arterial pressure and cardiac sympathetic tonus. Am J Physiol 273:H2738-H2745.

Chen CY, Bonham AC (2005) Glutamate suppresses GABA release via presynaptic metabotropic glutamate receptors at baroreceptor neurones in rats. J Physiol 562:535-551.

Chen CY, Munch PA, Quail AW, Bonham AC (2002) Postexercise hypotension in conscious SHR is attenuated by blockade of substance P receptors in NTS. Am J Physiol Heart Circ Physiol 283:H1856-H1862.

Collins HL, Rodenbaugh DW, DiCarlo SE (2001) Central blockade of vasopressin V(1) receptors attenuates postexercise hypotension. Am J Physiol Regul Integr Comp Physiol 281:R375-R380.

Degtyarenko AM, Kaufman MP (2005) MLR-induced inhibition of barosensory cells in the NTS. Am J Physiol Heart Circ Physiol 289:H2575-H2584.

Degtyarenko AM, Kaufman MP (2006) Barosensory cells in the nucleus tractus solitarius receive convergent input from group III muscle afferents and central command. Neuroscience 140:1041-1050.

Fitzgerald W (1981) Labile hypertension and jogging: new diagnostic tool or spurious discovery? Br Med J (Clin Res Ed) 282:542-544.

Kajekar R, Chen CY, Mutoh T, Bonham AC (2002) GABA(A) receptor activation at medullary sympathetic neurons contributes to postexercise hypotension. Am J Physiol Heart Circ Physiol 282:H1615-H1624.
Kenney MJ, Seals DR (1993) Postexercise hypotension. Key features, mechanisms, and clinical significance. Hypertension 22:653-664.

Marvizón JC, Wang X, Matsuka Y, Neubert JK, Spigelman I (2003) Relationship between capsaicin-evoked substance $\mathrm{P}$ release and neurokinin 1 receptor internalization in the rat spinal cord. Neuroscience 118:535-545.

Mifflin SW (2001) What does the brain know about blood pressure? News Physiol Sci 16:266-271.

Potts JT (2001) Exercise and sensory integration. Role of the nucleus tractus solitarius. Ann N Y Acad Sci 940:221-236.

Potts JT (2006) Inhibitory neurotransmission in the nucleus tractus solitarii: implications for baroreflex resetting during exercise. Exp Physio 91:59-72.

Potts JT, Fuchs IE, Li J, Leshnower B, Mitchell JH (1999) Skeletal muscle afferent fibres release substance $\mathrm{P}$ in the nucleus tractus solitarii of anaesthetized cats. J Physiol 514:829-841.

Potts JT, Paton JF, Mitchell JH, Garry MG, Kline G, Anguelov PT, Lee SM (2003) Contraction-sensitive skeletal muscle afferents inhibit arterial baroreceptor signalling in the nucleus of the solitary tract: role of intrinsic GABA interneurons. Neuroscience 119:201-214.

Rosamond W, Flegal K, Furie K, Go A, Greenlund K, Haase N, Hailpern SM, Ho M, Howard V, Kissela B, Kittner S, Lloyd-Jones D, McDermott M, Meigs J, Moy C, Nichol G, O’Donnell C, Roger V, Sorlie P, Steinberger J, et al. (2008) Heart disease and stroke statistics_-2008 update: a repor from the American Heart Association Statistics Committee and Stroke Statistics Subcommittee. Circulation 117:e25-e146.

Sekizawa S, Bonham AC (2006) Group I metabotropic glutamate receptors on second-order baroreceptor neurons are tonically activated and induce a Na+-Ca2+ exchange current. J Neurophysiol 95:882-892.

Toney GM, Mifflin SW (1995) Time-dependent inhibition of hindlimb somatic afferent transmission within nucleus tractus solitarius: an in vivo intracellular recording study. Neuroscience 68:445-453.

Tsukamoto K, Sved AF (1993) Enhanced gamma-aminobutyric acidmediated responses in nucleus tractus solitarius of hypertensive rats. Hypertension 22:819-825.

Yao T, Andersson S, Thoren P (1982) Long-lasting cardiovascular depressor response following sciatic stimulation in spontaneously hypertensive rats Evidence for the involvement of central endorphin and serotonin systems. Brain Res 244:295-303.

Zhang W, Herrera-Rosales M, Mifflin S (2007) Chronic hypertension enhances the postsynaptic effect of baclofen in the nucleus tractus solitarius. Hypertension 49:659-663. 